- Case Report

\title{
I Can Not Wear My Sunglasses: An Unusual Sarcoidosis Presentation
}

\author{
José Pablo Miramontes González ${ }^{1,2, *}$, Virginia Velasco Tirado ${ }^{2,3}$, Pablo González García ${ }^{1,2}$, Marta Sánchez García', \\ Ángeles Fidalgo Fernández ${ }^{1}$
}

'Department of Internal Medicine Unit, University Hospital Salamanca, Salamanca, Spain

${ }^{2}$ IBSAL, Institute Biomedical Research of Salamanca, Salamanca, Spain

${ }^{3}$ Department of Dermatology, University Hospital Salamanca, Salamanca, Spain

A 39-year-old Caucasian man was referred to University Hospital Salamanca from a primary care unit due to the presence of an erythematous violaceous nodule at the superior portion of his nose. Physical examination indicated that the firm, fixed erythematous violaceous nodule measured approximately $2 \mathrm{~cm}$ in diameter and was located inferior to a scar on the nasal bridge. Cutaneous involvement in sarcoidosis occurs in $25 \%$ of cases. A wide range of clinical presentations of cutaneous sarcoidosis is recognized. Skin lesions are classified as either non-specific, of which erythema nodosum is the most representative and specific, or as granulomatous, which includes maculopapular nodules, plaques, infiltrated scars, lupus pernio, ulcerations, warty lesions and erythroderma. Scar sarcoidosis is a type of cutaneous sarcoidosis.

Keywords: Cicatrix; Sarcoidosis; Cutaneous 


\section{INTRODUCTION}

Sarcoidosis is a granulomatous non-infectious disease with an unknown etiology and an ability to affect several organs, including the eyes, lymph nodules, bones, skin, and lungs. The most frequent sarcoidosis presentation involves the lungs and other internal organs. Sarcoidosis usually occurs in young adults between 25 and 35 years of age as well as between 45 and 55 years of age. There is a greater prevalence of the disease among women. ${ }^{1)}$ Cutaneous sarcoidosis is known as a 'great simulator' of other diseases due to its polymorphic lesion appearance ${ }^{2)}$ as well as its complex and varied range of clinical presentation. Meanwhile, cicatricial sarcoidosis is rare, does not always present in a manner that is characteristic of cutaneous sarcoidosis, and represents a major diagnostic challenge. ${ }^{3,4)}$ Therefore, clinical suspicion is necessary to correctly diagnose the condition.

\section{CASE REPORT}

A white male of 39 years of age was referred to University Hospital Salamanca from a primary care unit due to the presence of an erythematous violaceous nodule at the superior portion of his nose (Figure 1). The patient's medical records were unremarkable except for a facial trauma and nose fracture from twenty years previous. The nodule increased in size during the last four weeks, thereby resulting in complications while wearing sunglasses. When asked about difficulty in breathing, the patient indicated difficult only while participating in strenuous physical activity in the form of cycling. Physical examination indicated that the firm, fixed erythematous violaceous nodule measured approximately $2 \mathrm{~cm}$ in diameter and was located inferior to a scar on the nasal bridge. The remainder of the examination was normal without lymphadenopathies, organomegaly, or nodules.

Blood test indicated elevated levels of angiotensin converting enzyme (301 U/L; normal range, 27 to $68 \mathrm{U} / \mathrm{L}$ ), while the remaining parameters, including markers of autoimmunity, were within the normal

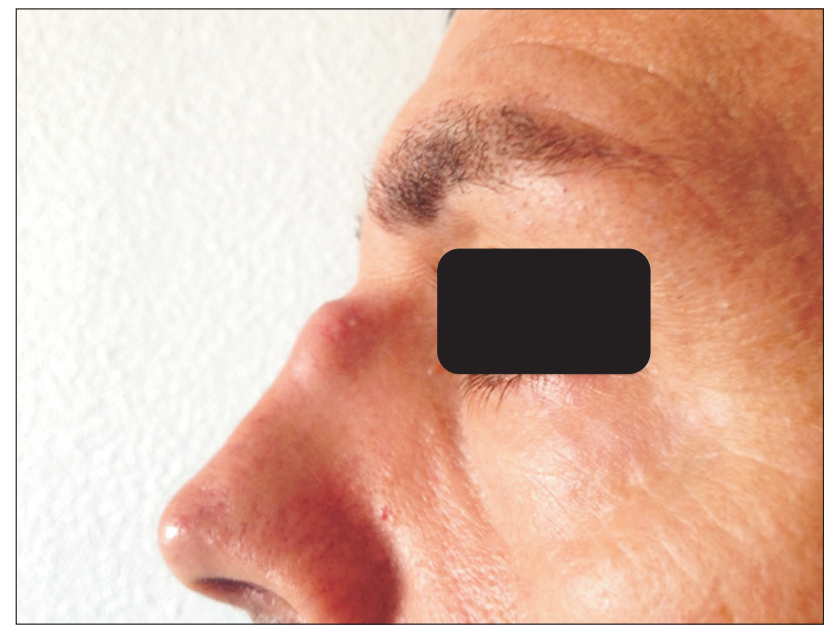

Figure 1. Lateral view of the nose showing a growing nodule on its superior portion. range. Chest radiograph showed an increase in size of the lung hilum, the presence of lymph nodes in the mediastinum and an interstitial pattern in both lung (Figure 2). Tomography confirmed the results of the chest radiograph, while the biopsy indicated the presence of confluent granulomas of epithelioid histiocytes that were mixed with multinucleated giant cells. These findings were compatible with sarcoidosis (Figure 3).

The patient received $0.05 \%$ topical clobetasol propionate twice daily, which resulted in an improvement in the skin lesion, and systemic corticosteroids (methylprednisolone).

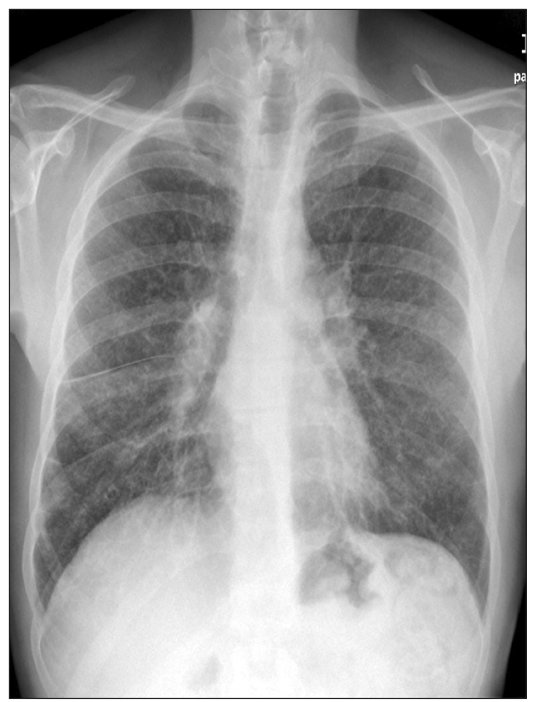

Figure 2. Chest radiograph showing an interstitial pattern and bilateral hilar enlargement with lymph nodes.

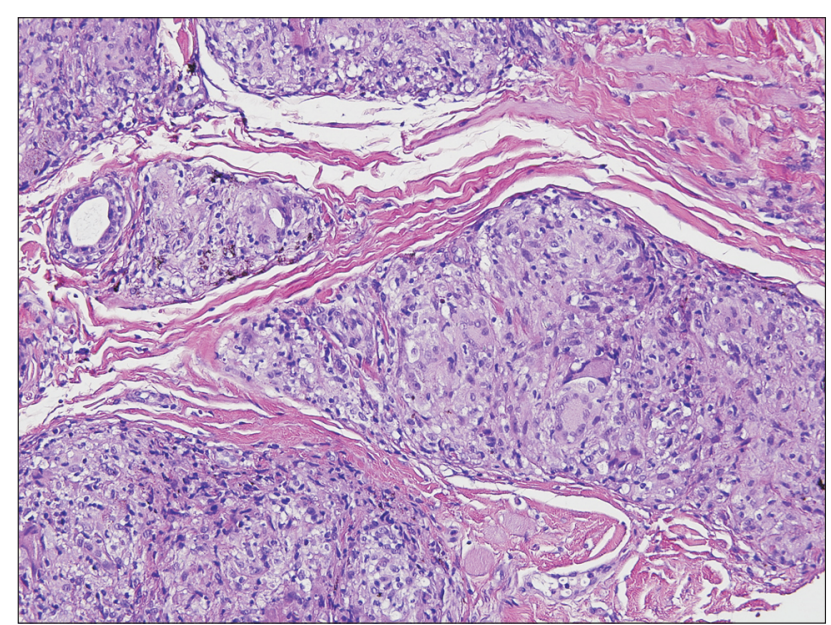

Figure 3. Pathological anatomy (H\&E, x100). Confluent granulomas of epithelioid histiocytes mixed with multinucleated giant cells, which indicates the possible presence of sarcoidosis. 


\section{DISCUSSION}

Sarcoidosis is a multisystem disorder whose cause is unknown. Nevertheless, it is characterized by the presence of a non-caseating inflammatory granuloma. Despite extensive research, the etiology of sarcoidosis has not yet been elucidated, although most evidence supports that the pathogenic mechanism of sarcoidosis is an aberrant immune response that is driven by an unidentified antigen (or antigens) in genetically susceptible individuals. ${ }^{5)}$ The incidence of sarcoidosis varies widely across different ethnic and racial groups around the world. The highest annual incidence of sarcoidosis has been observed in northern European countries (5 to 40 cases per 100,000 people). In Japan, the annual incidence ranges from 1 to 2 cases per 100,000 people and peaks in the third decade of life. In Korea, the incidence was less than 1 case per 100,000 people, with the most common symptoms being respiratory $(42 \%)$ in nature and the most frequently affected organs being the lungs (87\%) and the skin (31\%). The adjusted annual incidence among black Americans is roughly three times that of white Americans (35.5 cases per 100,000, as compared with 10.9 per 100,000). ${ }^{2,6-8)}$

Cutaneous involvement in sarcoidosis occurs in $25 \%$ of cases, with clinicians recognizing a wide range of presentations of cutaneous sarcoidosis. Skin lesions are classified as either non-specific, of which erythema nodosum is the most representative and specific, or as granulomatous, which includes maculopapular nodules, plaques, infiltrated scars, lupus pernio, ulcerations, warty lesions, and erythroderma. Recognizing cutaneous lesions is important because, when present, these lesions serve as an easily accessible source of tissue for examination (skin biopsy), thereby avoiding more invasive procedures. Infiltration of cutaneous scars by non-caseating epithelioid cell granulomas is the hallmark epidermal characteristic, but an uncommon cutaneous manifestation, of sarcoidosis. This condition is known as scar sarcoidosis. Is the characteristic of the disease. Most patients with scar sarcoidosis have other systemic manifestations, particularly changes to the lungs. ${ }^{2,4,9)}$

In addition to the reactivation of scars that developed following pre- vious wounds, scar sarcoidosis has been reported at the sites of previous intramuscular injections, blood donation puncture, tattoo, and herpes zoster; in regions damaged during ritual sacrifice; and at the sites of allergen extract placement as a form of desensitization treatment and following hyaluronic acid injection and laser surgery. In general, scar sarcoidosis involves different skin levels. In most cases, the superficial and deep dermis is involved. Incision biopsy is needed to provide a diagnosis. Further investigations of systemic involvement in scar sarcoidosis should be performed in future studies. ${ }^{10)}$

\section{CONFLICT OF INTEREST}

No potential conflict of interest relevant to this article was reported.

\section{REFERENCES}

1. Fernandez-Faith E, McDonnell J. Cutaneous sarcoidosis: differential diagnosis. Clin Dermatol 2007;25:276-87.

2. Rybicki BA, Major M, Popovich J Jr, Maliarik MJ, Iannuzzi MC. Racial differences in sarcoidosis incidence: a 5-year study in a health maintenance organization. Am J Epidemiol 1997;145:234-41.

3. Wu JJ, Schiff KR. Sarcoidosis. Am Fam Physician 2004;70:312-22.

4. Hong YC, Na DJ, Han SH, Lee YD, Cho YS, Han MS. A case of scar sarcoidosis. Korean J Intern Med 2008;23:213-5.

5. Saidha S, Sotirchos ES, Eckstein C. Etiology of sarcoidosis: does infection play a role? Yale J Biol Med 2012;85:133-41.

6. Pietinalho A, Hiraga Y, Hosoda Y, Lofroos AB, Yamaguchi M, Selroos O. The frequency of sarcoidosis in Finland and Hokkaido, Japan: a comparative epidemiological study. Sarcoidosis 1995;12:61-7.

7. Kim DS. Sarcoidosis in Korea: report of the Second Nationwide Survey. Sarcoidosis Vasc Diffuse Lung Dis 2001;18:176-80.

8. Kim YJ, Kim YD. A case of scar sarcoidosis of the eyelid. Korean J Ophthalmol 2006;20:238-40.

9. Mantese SA, Berbert AL, Cesario TS, Silva HB. Sarcoidosis on skin scars: a case report. An Bras Dermatol 2010;85:903-5.

10. Singal A, Vij A, Pandhi D. Post herpes-zoster scar sarcoidosis with pulmonary involvement. Indian Dermatol Online J 2014;5:77-9. 